\title{
A Family with Myasthenia Gravis With and Without Thymoma
}

\author{
DL. Rotstein, V. Bril
}

Can J Neurol Sci. 2012; 39: 539-540

Acquired myasthenia gravis (MG) is an autoimmune condition targeting the neuromuscular junction. Thymomas have been found in $10-15 \%$ of patients with acquired MG suggesting a paraneoplastic origin in these cases, and approximately onethird are designated as malignant with invasion of the capsule. Patients with thymoma are slightly older and more often have generalized symptoms compared to MG patients without thymoma. ${ }^{1}$ Although familial autoimmune $\mathrm{MG}$ has been reported occasionally, there is only one reported case of familial paraneoplastic MG from thymoma. ${ }^{2}$ Here we report a case of two siblings with paraneoplastic MG, where histological similitude was demonstrated by immunophenotyping. Their mother had earlier been diagnosed with MG, but had no evidence of thymoma. This is the first case reported of MG with thymoma and similar immunophenotyping in two or more family members, coexisting with MG without thymoma in the same family.

\section{CASE}

A 43-year-old Iranian woman presented with bilateral ptosis and diplopia that improved with rest. She denied bulbar symptoms and limb weakness. She had a five year history of episodic retrosternal chest pain. Examination showed asymmetrical ptosis at rest, limitation of adduction and abduction of the eyes, worse on the right side, and mild bifacial weakness. Power was normal in all other groups. She was graded Class I on the MGFA Clinical Classification scheme. Single fibre electromyography (EMG) showed increased jitter and abnormal pairs. The acetylcholine receptor antibody titre was elevated at $8.0 \mathrm{nM}$. Computed tomogram (CT) chest revealed an anterior mid-sternal mass suspicious for thymoma. Surgical exploration confirmed the thymoma and showed invasion of the right upper lobe of the lung. Histology demonstrated a World Health Organization (WHO) type B2 thymoma. Immunophenotyping of the tumour was positive for CD3 and pankeratin AE1/3. She was treated with pyridostigmine, prednisone and radiotherapy with complete resolution of her ocular symptoms.

The patient has a younger brother who developed ptosis, diplopia, facial weakness, and limb weakness at age 24, with an MGFA Class IIIa. Single fibre EMG showed increased jitter. The CT chest revealed a thymoma confirmed by needle biopsy. He received preoperative chemotherapy followed by surgical resection which showed an invasive thymoma with involvement of the pericardium and right upper lobe of the lung. Histology showed a WHO grade type B2 tumour, positive for CD3 and AE1/3 immunhistochemistry. The brother was treated with postoperative radiotherapy and pyridostigmine that was slowly tapered. The patient remains in full remission from $\mathrm{MG}$ and without evidence of tumour recurrence since 2002.

The mother of these two siblings also had a history of MG. She had developed diplopia and ptosis in her fifties (MGFA Class I). Her CT scan, performed in Iran, did not show thymoma. Unfortunately, the patient's mother and brother were residing in other countries and we were unable to ascertain their acetylcholine receptor antibody status from the medical records obtained. The mother's symptoms resolved nearly completely with pyridostigmine and corticosteroids. The patient's mother has eleven siblings, none of whom ever had symptoms or signs of myasthenia. A third sibling of the two with thymoma is well without MG. The patient's parents are not consanguineous. The father of the affected sibling pair never had symptoms of myasthenia. There was no family history of other autoimmune diseases.

\section{DISCUSSION}

This is the first reported case with immunophenotypic evidence of histologically identical thymomas associated with MG in first degree relatives. Paraneoplastic MG is usually associated with type B thymomas as found in these individuals. There is one previously reported case of thymoma and myasthenia gravis in two brothers from 1986. ${ }^{2}$ The brothers presented at ages 31 and 42 and met clinical, laboratory, and electrophysiologic criteria for MG. Both brothers had thymomas with epitheliod (type B) histology, but detailed phenotyping was not available. The authors in that case wondered whether the coexistence of MG and thymoma in the same sibling pair could be a chance occurrence, and even calculated the likelihood of this intersection as one in $4 \times 10^{10}$. Another case has since been reported of familial thymoma, but without MG, associated with a constitutional chromosome translocation $\mathrm{t}(14 ; 20)(\mathrm{q} 24 ; \mathrm{p} 12) .^{3}$ The family manifested various autoimmune disorders, including DM I, Sjogren's syndrome, Grave's disease, and pernicious anemia, but not MG.

The majority of cases of familial MG demonstrate heterogeneity in the clinical expression of MG. In some reported

From the Division of Neurology, Department of Medicine, University Health Network, University of Toronto, Toronto, Ontario, Canada.

Received November 21, 2011. Final Revisions Submitted January 18, 2012. Correspondence to: Vera Bril, The Toronto General Hospital, 5 Eaton Centre Wing, Room 309, 200 Elizabeth Street, Toronto, Ontario, M5G 2C4, Canada.

Email: vera.bril@uhn.ca 
cases, autoantibody specificity differs. ${ }^{4}$ In others, MG has occurred in single individuals with and without thymoma in the same family. ${ }^{5}$ Previous authors have concluded that familial MG cannot be linked to a single HLA haplotype..$^{4-5}$

Myasthenia gravis, with or without thymoma, is generally not considered to be a hereditary condition. Exceptions highlight the possibility of genetic factors that may contribute to the development of MG. The finding of histologically similar thymomas in this sibling pair with MG increases the probability of an underlying genetic factor, rather than a chance occurrence as proposed by Pascuzzi et al. ${ }^{2}$ Furthermore the history of MG without thymoma in the same kindred implies that there may be multiple genetic and environmental factors interacting to account for the expression of the disease and these factors deserve investigation.

\section{REFERENCES}

1. Bril V, Kojic J, Dhanani A. The long-term clinical outcome of myasthenia gravis in patients with thymoma. Neurology. 1998; 51:1198-1200.

2. Pascuzzi RM, Sermas A, Phillips LH, Johns TR. Familial autoimmune myasthenia gravis and thymoma: occurrence in two brothers. Neurology. 1986;36(3):423-7.

3. Nicodeme F, Geffroy S, Conti M, et al. Familial occurrence of thymoma and autoimmune diseases with the constitutional translocation $\mathrm{t}(14 ; 20)(\mathrm{q} 24.1 ; \mathrm{p} 12.3)$. Genes Chromosomes Cancer. 2005;44(2):154-60.

4. Lavrnic D, Nikolic A, De Baets M, et al. Familail occurrence of autoimmune myasthenia gravis with different antibody specificity. Neurology. 2008;70(21):2011-3.

5. Evoli A, Batocchi AP, Zelano G, Uncini A, Palmisani MT, Tonali P. Familial autoimmune myasthenia gravis: report of four families. J Neurol Neurosurg Psych. 1995;58:729-31. 